\title{
Countable sections for locally compact group actions
}

\author{
ALEXANDER S. KECHRIS $\dagger$ \\ Department of Mathematics, California Institute of Technology, Pasadena, \\ CA 91125, USA
}

(Received 1 March 1991)

\begin{abstract}
It has been shown by J. Feldman, P. Hahn and C. C. Moore that every non-singular action of a second countable locally compact group has a countable (in fact so-called lacunary) complete measurable section. This is extended here to the purely Borel theoretic category, consisting of a Borel action of such a group on an analytic Borel space (without any measure). Characterizations of when an arbitrary Borel equivalence relation admits a countable complete Borel section are also established.
\end{abstract}

\section{Introduction}

(I) The purpose of this paper is to provide a purely Borel-theoretic extension of the main result of Feldman-Hahn-Moore [FHM] concerning countable and socalled lacunary complete sections in non-singular actions of second countable locally compact groups. Let us introduce first the relevant definitions.

If $E$ is an equivalence relation on a set $X$, a countable section for $E$ is a set $Y \subseteq X$ such that card $\left(Y \cap[x]_{E}\right) \leq \aleph_{0}$, for each $E$-equivalence class $[x]_{E}$. Such a section is complete if it meets every equivalence class. If $G$ is a topological group acting on a space $X$ and $E_{G}$ is the equivalence relation induced by the orbits of this action, then a set $Y \subseteq X$ is called a lacunary section if there is a neighborhood $U$ of the identity of $G$ such that for all $y \in Y, y U \cap Y=\{y\}$ (we write $y U=\{y g: g \in U\}$, where $(y, g) \mapsto y g$ is the action). A complete lacunary section is defined analogously. It is easy to see that if $G$ is second countable, any lacunary section is countable.

TheOREM 1.1. Let $G$ be a second countable locally compact group and $X$ a Polish space on which $G$ acts continuously (i.e. the map $(x, g) \in X \times G \mapsto x g \in X$ is continuous). Let $E_{G}$ be the induced equivalence relation (i.e. $x E_{G} y \Leftrightarrow \exists g \in G(x=y g)$ ). Then $E_{G}$ has a complete lacunary Borel section. In particular, $E_{G}$ has a complete countable Borel section.

By a result of Varadarajan [Var], for each second countable locally compact group $G$ there is a compact metric space $\tilde{G}$ and a continuous action $\alpha$ of $G$ on $\tilde{G}$ with the following universal property: If $X$ is a standard (resp. analytic) Borel space 
and $\beta$ a Borel action of $G$ on $X$ (i.e. $(x, g) \in X \times G \rightarrow \beta(x, g)=x g \in X$ is Borel), there is an $\alpha$-invariant Borel (resp. analytic) set $\hat{X} \subseteq \tilde{G}$ so that the action $\beta$ is Borel isomorphic to the restriction of $\alpha$ to $\hat{X}$. We have therefore the following

COROLLARY 1.2. Let $G$ be a second countable locally compact group and $X$ an analytic Borel space on which $G$ acts in a Borel way. Let $E_{G}$ be the induced equivalence relation. Then $E_{G}$ has a complete lacunary (therefore countable) Borel section.

The result of Feldman-Hahn-Moore, referred to earlier, asserts that in the context of Corollary 1.2, if $\mu$ is a probability measure on $X$ which is $G$-quasi-invariant, i.e. $\mu \sim \mu g$ for any $g \in G$, then there is a Borel lacunary section $B$ with $[B]_{E_{G}}=$ $\left\{x: \exists y \in B\left(x E_{G} y\right)\right\}$ of $\mu$-measure 1. It appears that the proof in [FHM] of this measure theoretic version could be suitably modified to prove the existence of just countable complete Borel sections in the pure Borel context of Corollary 1.2. However, the proof in [FHM] uses the deep structure theory of locally compact groups (see $[\mathbf{M Z}]$ ), which allows in [FHM] the reduction to the case of Lie groups, where special tools are available. On the other hand, our proof of Theorem 1.1 is purely descriptive set theoretic and uses only the definition of locally compact groups. (The result of Varadarajan [Var] used to derive Corollary 1.2 needs further only the existence of Haar measure. Added in proof. We have recently found a proof of Varadarajan's theorem which also avoids Haar measure.) Another proof of the Feldman-Hahn-Moore theorem has been given in Ramsay [R1], which actually applies to more general situations, but still in the measure theoretic category. We do not know if Ramsay's method can be used to produce pure Borel theoretic results. We will discuss (the relevant to us form of) Ramsay's theorem and a related open problem concerning an extension of Theorem 1.1 in IV) below. In Wagh [W], the author proves the special case of Theorem 1.1 for $G=\mathbb{R}$, using density arguments on $\mathbb{R}$ (see also [R2, § 4]). This is the Borel version of the result in Ambrose [A]. As in [FHM], we also make use of an idea of Forrest [F]. Finally we draw some inspiration from the result of Burgess [B] and thus ultimately from Vaught [Vau].

(II) We have recently been interested in the study of the structure of Borel equivalence relations (see for example [HKL, Ke2]) on Polish (equivalently standard Borel) spaces. A partial (pre)order is introduced which measures the relative complexity of Borel equivalence relations. For $E, F$ Borel equivalence relations on $X$, $Y$ resp. we say that $E$ is reducible to $F$, in symbols $E \leq F$, if there is a Borel map $f: X \rightarrow Y$ with $x E x^{\prime} \Leftrightarrow f(x) F f\left(x^{\prime}\right)$. If an injective such $f$ can be found we say that $E$ is embeddable in $F$, in symbols $E \subseteq F$. Finally let

$$
\begin{gathered}
E \approx * F \Leftrightarrow E \leq F \wedge F \leq E \\
E \approx F \Leftrightarrow E \sqsubseteq F \wedge F \sqsubseteq E .
\end{gathered}
$$

Among the class of Borel equivalence relations the subclass of so-called countable ones has received special attention. An equivalence relation is called countable if every equivalence class is countable. By a result of Feldman-Moore $[F M]$, the countable Borel equivalence relations are exactly those induced by Borel actions of countable groups. The following immediate corollary of 1.2 shows that up to 
$\approx *$-equivalence the class of countable Borel equivalence relations includes the ones induced by Borel actions of second countable locally compact groups.

Corollary 1.3. Let $G$ be a second countable locally compact group and $X$ a standard Borel space on which $G$ acts in a Borel way. Let $E_{G}$ be the induced equivalence relation. Then there is a countable Borel equivalence relation $F$ such that $E_{G} \approx{ }^{*} F$.

More information can be obtained for $G=\mathbb{R}$. Recall that a Borel equivalence relation $E$ on $X$ is called hyperfinite if $E$ is induced by a Borel action of $\mathbb{Z}$ (i.e. $E$ is induced by the orbits of a single Borel automorphism).

Corollary 1.4. (Wagh [W].) In the notation of 1.3 , if $G=\mathbb{R}, F$ can be taken to be hyperfinite.

It would be interesting to characterize the class of $E$ which are reducible to countable Borel $F$. It is not true that all such $E$ are induced by Borel actions of second countable locally compact groups (we will see a simple example in $\S 2$ ).

There is however a somewhat interesting characterization of those Borel equivalence relations that admit complete countable Borel sections, that comes out of the proof of Theorem 1.1. We need some definitions first.

Let $E$ be a Borel equivalence relation on $X$. Denote by $X / E$ the quotient space and by $C$ a typical equivalence class of $E$. Let $C \in X / E \mapsto I_{C}$ be a map assigning to each $C$ a $\sigma$-ideal of subsets of $C, I_{C}$, with $C \notin I_{C}$. We say that $C \mapsto I_{C}$ is Borel if for each Borel set $A \subseteq X^{2}$ the set $A_{l}$ defined by

$$
x \in A_{I} \Leftrightarrow\left\{y \in[x]_{E}: A(x, y)\right\} \in I_{[x]_{E}}
$$

is Borel. Finally, we say that $C \mapsto I_{C}$ has the ccc (countable chain condition) if every $I_{C}$ has the ccc. (A $\sigma$-ideal $I$ of subsets of a set $A$ has the ccc if any collection of pairwise disjoint subsets of $A$ which are not in $I$ is countable.)

Here are some examples of Borel ccc assignments:

(i) Let $E$ be a Borel equivalence relation on $X$ induced by a Borel action of a Polish group $G$, i.e. $E=E_{G}$. Given $C \in X / E$, put for $A \subseteq C$

$$
A \in I_{C} \Leftrightarrow\{g: x g \in A\} \text { is meager (in } G \text { ) }
$$

where $x \in C$. It is easy to see that this is well defined independently of $x \in C$. To verify that $C \mapsto I_{C}$ is Borel, note that for $A \subseteq X^{2}$

$$
x \in A_{I} \Leftrightarrow\left\{y \in[x]_{E}: A(x, y)\right\} \in I_{[x]_{E}} \Leftrightarrow\{g: A(x, x g)\} \text { is meager }
$$

so this follows from standard facts about Borel definability of category notions (see $\S 2$ ). Moreover, $I_{C}$ has the ccc as the $\sigma$-ideal of meager sets in $G$ has the ccc.

(ii) Let now $E, G$ be as in (i) but additionally with $G$ locally compact. Let $\mu$ be Haar measure on $G$. For each $C \in X / E$ and $x \in C$ let $f_{x}: G \rightarrow[x]_{E}$ be defined by $f_{x}(g)=x g$ and let $\mu_{x}=f_{x \mu}$, the image of $\mu$ under $f_{x}$. Let, for $A \subseteq C$,

$$
A \in I_{C} \Leftrightarrow \mu_{x}(A)=0 \Leftrightarrow \mu(\{g: x g \in A\})=0 .
$$

Again this is independent of $x$, Borel (by similar results on Borel definability of measure theoretic notions) and has the ccc. 
(ii) Generalizing (ii), let now $E$ be a Borel equivalence relation on $X$ and let $x \in X \mapsto \mu_{x}^{*} \in P(X)$, be a Borel map from $X$ into the (standard) Borel space of probability measures on $X$, such that $\mu_{x}^{*}\left([x]_{E}\right)=1$ and $x E y \Rightarrow \mu_{x}^{*} \sim \mu_{y}^{*}$. Put for $C \in X / E, x \in C$

$$
A \in I_{C} \Leftrightarrow \mu_{x}^{*}(A)=0 .
$$

Again this works. To see that this indeed generalizes (ii), note that if $\mu(G)=1$ we can take $\mu_{x}^{*}=\mu_{x}$. If $\mu(G)=\infty$, let $F_{n}$ be Borel pairwise disjoint with $G=\bigcup_{n} F_{n}$ and $\mu\left(F_{n}\right)=1$. Put then

$$
\mu_{x}^{n}(A)=\mu\left(F_{n} \cap\{g: x g \in A\}\right)
$$

and $\mu_{x}^{*}=\sum 1 / 2^{n+1} \cdot \mu_{x}^{n}$.

Recall that a Borel equivalence relation $E$ on $X$ is smooth if there is Borel $f: X \rightarrow Y, Y$ Polish with $x E x^{\prime} \Leftrightarrow f(x)=f\left(x^{\prime}\right)$.

THEOREM 1.5. Let $E$ be a Borel equivalence relation on $X$. Then the following are equivalent:

(i) E has a complete countable Borel section.

(ii) (a) $E=\bigcup_{n} E_{n}$, where each $E_{n}$ is a smooth Borel equivalence relation, and

(b) There is a Borel ccc assignment $C \in X / E \mapsto I_{C}$.

(iii) As in (ii) but with (b) replaced by

(b') There is a Borel assignment $x \mapsto \mu_{x}$ with $\mu_{x}\left([x]_{E}\right)=1, x E y \Rightarrow \mu_{x} \sim \mu_{y}$.

Both conditions in (ii) are necessary, but we do not know if in (iii) condition (a) is needed. We will discuss some relevant examples and open problems in (III), (IV) below.

(III) Suppose now $G$ is a standard Borel group, i.e. $G$ has a standard Borel structure and the group operations are Borel. Then it is well known that there is at most one Polish topology with the same Borel structure under which $G$ becomes a topological group. If such a topology exists we call (by abuse of language) $G$ itself Polish. If moreover this topology is locally compact we call $G$ Polish locally compact (i.e. second countable locally compact). For certain standard Borel groups we can provide a characterization of when they are Polish locally compact, which can be viewed as a kind of converse of Theorem 1.1. We do not know if the full converse is true for arbitrary standard Borel groups. We would like to thank the referee for suggesting the formulation of the hypothesis of the next result (our original one was more restrictive) and for pointing out that (iv) $\Rightarrow$ (i), in a somewhat stronger form, is contained in Theorem A of [FR]. The referee also pointed out that the example of the equivalence relation $E_{1}$ below is also discussed in this paper.

THEOREM 1.6. Let $G$ be a standard Borel group and assume that $G$ admits a Borel action $(g, x) \mapsto x g$ on a standard Borel space $X_{0}$, which is free (i.e., $g \neq 1, x \in X_{0} \Rightarrow x g \neq$ $x$ ) and has a quasi-invariant probability measure $\mu$ (i.e., $\mu \sim \mu g$, for all $g \in G$.) Then the following are equivalent:

(i) $G$ is Polish locally compact.

(ii) For every Borel action of $G$ on a standard Borel space $X$ there is a complete countable Borel section for $E_{G}$. 
(iii) For every Borel action of $G$ on a standard Borel space $X, E_{G}$ is reducible to a countable Borel equivalence relation.

(iv) For the action of $G$ on $X_{0}$ given in the hypothesis and denoting by $E_{G}^{0}$ the corresponding equivalence relation, there is a complete countable Borel section for $E_{G}^{0}$.

(v) For $E_{G}^{0}$ as in (iv), $E_{G}^{0}$ is reducible to a countable Borel equivalence relation.

The hypothesis of 1.6 is true if $G$ is a Borel subgroup of a Polish locally compact group $H$. In this case $X_{0}=H, G$ acts on $H$ by right multiplication and $\mu$ is a probability measure equivalent to Haar measure on $H$. The referee raised the question of whether any group satisfying the hypothesis of 1.6 is a Borel subgroup of a Polish locally compact group.

For example, it follows immediately from this result that if $X=\pi^{N}$, where $\pi$ is the unit circle, and $E_{1}$ is defined by

$$
\left(x_{n}\right) E_{1}\left(y_{n}\right) \Leftrightarrow \exists m \forall n \geq m\left(x_{n}=y_{n}\right)
$$

then $E_{1}$ is not reducible to a countable Borel equivalence relation. Since $E_{1}$ is clearly (an increasing in fact) union of a sequence of smooth Borel equivalence relations, this shows that some condition beyond (a) is needed in (ii), (iii) of Theorem 1.5. (That $E_{1}$ is not reducible to a countable Borel equivalence relation has been known in some form or other for some time in ergodic theory-see [FHM]-and has been also proved using category methods by Jackson and Louveau independently).

We also use Theorem 1.6 in $\S 5$ to show that there is an example of a $K_{\sigma}$ equivalence relation on $\mathbb{T}^{N}$ induced by a free continuous action of a Polish group which is not again reducible to a countable Borel equivalence relation. This shows for instance that condition (b) is not enough in Theorem 1.5.

Remark. The remark after Theorem 1.6 should be compared with the following result of Mackey [Ma] and Miller [Mi]: let $H$ be a Polish group and $G \subseteq H$ a Borel subgroup. If $E_{G}$ is the equivalence relation induced by the action of $G$ on $H$ by right multiplication, then the following are equivalent:

(i) $G$ is closed,

(ii) $E_{G}$ has a Borel transversal,

(iii) $E_{G}$ is smooth.

(IV) We discuss now some further open problems.

The result of Ramsay [R1] alluded in (I) asserts the following: let $E$ be a Borel equivalence relation on $X$ and assume there is a Borel assignment of probability measures $x \mapsto \mu_{x}$, so that $\mu_{x}\left([x]_{E}\right)=1$ and $x E y \Rightarrow \mu_{x} \sim \mu_{y}$. If $\mu$ is a probability measure on $X$, then there is a Borel set $B \subseteq X$ such that for $\mu$-almost all $x$, $B \cap[x]_{E} \neq \varnothing$ and card $\left({ }_{B} \cap[x]_{E}\right) \leq \aleph_{0}$.

It would be interesting to find a Borel theoretic version of this type of result. One possible formulation is the following: Let $E$ be a $K_{\sigma}$ equivalence relation on a Polish compact space $X$ and assume that there is a Borel assignment of probability measures $x \mapsto \mu_{x}$, so that $\mu_{x}\left([x]_{E}\right)=1$ and $x E y \Rightarrow \mu_{x} \sim \mu_{y}$. Then $E$ has a complete countable Borel section. 
We have restricted ourselves to $K_{\sigma}$ relations as these are more manageable (and by measure-theoretic approximations a positive answer even in this case would imply Ramsay's Theorem). However, we do not know any obstruction to a more general result for arbitrary Borel $E$. We do want to point out though that one could not hope for further generalizations, where the assignment $x \mapsto \mu_{x}$ is replaced by a Borel ccc assignment $C \mapsto I_{C}$, in view of the example mentioned at the end of (III).

(V) The rest of this paper is organized as follows: in $\S 2$ we prove Theorem 1.1, in $\S 3$ Theorem 1.5 , in $\S 4$ Theorem 1.6 and in $\S 5$ we discuss the examples mentioned in (III).

\section{Proof of Theorem 1.1}

Fix a metric $d$ on $X$. Fix also a compact nbhd $\Lambda$ of $1 \in G$ and a compact symmetric $\left(\Delta=\Delta^{-1}\right)$ nbhd of 1 such that $\Delta^{2} \subseteq \Lambda$.

Consider the following relation on $X$ :

$$
R(x, y) \Leftrightarrow \exists g \in \Delta(y=x g) .
$$

Following Forrest [F] and Feldman-Hahn-Moore [FHM], we will find a sequence $X_{n} \subseteq X$ such that $X=\bigcup_{n} X_{n}$ and $R \mid X_{n}\left(=R \cap X_{n}^{2}\right)$ is an equivalence relation (on $\left.X_{n}\right)$. To do this, for each $\varepsilon>0$ put

$$
A_{\varepsilon}=\left\{x \in X: \forall g \in G\left[(d(x, x g) \leq \varepsilon \wedge g \in \Lambda) \Rightarrow g \in G_{x} \Delta^{0}\right]\right\}
$$

where $G_{x}=\{g: x g=x\}$ is the stabilizer of $x$ and $\Delta^{0}=$ int $(\Delta)$.

Claim 1. $X=\bigcup_{n \geq 1} A_{1 / n}$.

Proof. Fix $x \in X$. If $x \notin \bigcup_{n \geq 1} A_{1 / n}$, towards a contradiction, find for each $n \geq 1$, $g_{n} \in G$ with $d\left(x, x g_{n}\right) \leq 1 / n$ and $g_{n} \in \Lambda-G_{x} \Delta^{0}$. Since $\Lambda-G_{x} \Delta^{0}$ is compact, by going to a subsequence, we can assume that $g_{n} \rightarrow g \in \Lambda-G_{x} \Delta^{0}$. As the action is continuous and $d\left(x, x g_{n}\right) \leq 1 / n$, it follows that $d(x, x g)=0$, i.e. $g \in G_{x}$ so that $g \in G_{x} \Delta^{0}$, a contradiction.

Claim 2. Let $B \subseteq A_{\varepsilon}$, $\operatorname{diam}(B) \leq \varepsilon$. Then $R \mid B$ is an equivalence relation.

Proof. As $\Delta=\Delta^{-1}$ and $1 \in \Delta, R$ is reflexive and symmetric. Fix now $x, y, z \in B$ with $R(x, y), R(y, z)$. Let $g, h \in \Delta$ be such that $y=x g, z=y h$. Then $z=x g h, g h \in \Delta^{2} \subseteq \Lambda$ and $d(x, x g h) \leq \varepsilon$, so, as $x \in A_{\varepsilon}, g h \in G_{x} \Delta^{0}$, i.e. $g h=p q$ with $p \in G_{x}, q \in \Delta^{0}$. Then $z=x p q=x q$, i.e. $R(x, z)$.

By the two preceding claims, we can easily write $X=\bigcup_{n} X_{n}$, where each $X_{n}$ is of the form $A_{\varepsilon} \cap B$ for some ball $B$ of diameter $\leq \varepsilon$ and $R \mid X_{n}$ is an equivalence relation.

We will verify now that each $X_{n}$ is Borel. For that we will use the following classical result of descriptive set theory. (For this and other standard facts of descriptive set theory that we use later we refer the reader to Moschovakis [Mo].)

Theorem 2.1. If $\mathscr{X}, \mathscr{Y}$ are Polish spaces, $P \subseteq \mathscr{X} \times \mathscr{Y}$ is Borel and each section $P_{x}=$ $\{y: P(x, y)\}$ is $K_{c}$, then $\operatorname{proj}[P]=\{x: \exists y P(x, y)\} \subseteq \mathscr{X}$ is Borel.

To show that each $X_{n}$ is Borel it is enough to prove that each $A_{\varepsilon}$ is Borel.

Claim 3. $A_{f}$ is Borel. 
Proof. We have

$$
x \notin A_{\varepsilon} \Leftrightarrow \exists g P(x, g)
$$

where

$$
P(x, g) \Leftrightarrow d(x, x g) \leq \varepsilon \wedge g \in \Lambda \wedge g \notin G_{x} \Delta^{0} .
$$

So $P \subseteq X \times G, X-A_{\varepsilon}=\operatorname{proj}[P]$. It is enough therefore to check that $P$ is Borel and each section $P_{x} \subseteq G$ is $K_{\sigma}$ or, as $G$ is itself is $K_{\sigma}$, that $P_{x}$ is closed. The last statement is straightforward, so let us verify that $P$ is Borel. The conditions $d(x, x g) \leq$ $\varepsilon, g \in \Lambda$ are clearly closed, so it is enough to check that

$$
P^{\prime}(x, g) \Leftrightarrow g \in G_{x} \Delta^{0} \Leftrightarrow \exists h \exists p\left[x h=x \wedge p \in \Delta^{0} \wedge g=h p\right]
$$

is Borel. But $P^{\prime}$ is the projection of a Borel set $P^{\prime \prime}$ in $(X \times G) \times G^{2}$ whose sections $P_{x, g}^{\prime \prime} \subseteq G^{2}$ are $F_{\sigma}$ and thus, as $G^{2}$ is $K_{\sigma}$, actually $K_{\sigma}$.

Our next step is to show that $R \mid X_{n}$ is smooth. This will be based on the following standard fact.

Proposition 2.2. If $E$ is a closed equivalence relation on a Polish space $\mathscr{X}$, then $E$ is smooth.

Proof. If $(x, y) \notin E$, let $A, B$ be basic open sets in $\mathscr{X}$ with $(x, y) \in A \times B,(A \times B) \cap E=$ $\varnothing$. Then $[A]_{E} \cap[B]_{E}=\varnothing$ and $[A]_{E},[B]_{E}$ are analytic (here $[A]_{E}$ is the $E$-saturation of $A$.) Inductively define: $A_{0}=[A]_{E}, A_{1}=$ a Borel set separating $\left[A_{0}\right]_{E},[B]_{E}$ (i.e. $\left.A_{1} \supseteq[A]_{E}, A_{1} \cap[B]_{E}=\varnothing\right), A_{2}=\left[A_{1}\right]_{E}, A_{3}=$ a Borel set separating $\left[A_{2}\right]_{E},[B]_{E}$, etc. Let $A_{\infty}=\bigcup_{n} A_{n}$. Then $A_{\infty}$ is Borel, $E$-invariant and $x \in A_{\infty}, y \notin A_{\infty}$. Since there are only countably many such $A_{\infty}$ (choosing our $A, B$ from a fixed countable basis) it follows that there is a countable family of Borel sets $C_{n}$ with $x E y \Leftrightarrow$ $\forall n\left[x \in C_{n} \Leftrightarrow y \in C_{n}\right]$. But this is exactly smoothness of $E$.

Remark. In [HKL] it is shown that the preceding proposition is valid even for $G_{\delta} E$.

To show now that $R \mid X_{n}$ is smooth, note first that $R$ is closed (in $X^{2}$ ). Since $X_{n}$ is Borel, we can find a Polish topology $\tau$ on $X$ which extends its underlying topology but has no more Borel sets, such that $X_{n}$ becomes clopen in $\tau$ (see e.g. [Ku]). Look at the Polish space $\left(X_{n}, \tau\right)$. Since $R$ is closed in $X^{2}$, it is also closed in $\left(X^{2}, \tau \times \tau\right)$, so $R \mid X_{n}$ is a closed equivalence relation in the Polish space $X_{n}$ with the relativized from $\tau$ topology. By the preceding proposition $R \mid X_{n}$ is smooth.

To summarize: we have written $X=\bigcup_{n} X_{n}$, with $X_{n}$ Borel such that $R \mid X_{n}$ is smooth. Since $E:=E_{G}$ is induced by the Borel action of a Polish group, we can assign, as in example (i) of $\S 1$ (II), a $\sigma$-ideal $J_{C}$ to each $C \in X / E$ by

$$
B \in J_{C} \Leftrightarrow\{g \in G: x g \in B\} \text { is meager }
$$

(for any $B \subseteq C, x \in C$ ). Using this define for each $x \in X$,

$$
n(x)=\text { least } n \text { such that } X_{n} \cap[x]_{E} \notin J_{[x]_{E}} \text {. }
$$

Clearly $n(x)$ exists, as $[x]_{E} \notin J_{[x]_{E}}$ and $[x]_{E}=\bigcup_{n}\left(X_{n} \cap[x]_{E}\right)$ and depends only on $[x]_{E}$. Put

$$
x \in \tilde{Y} \Leftrightarrow x \in X_{n(x)}
$$

We will verify that $\tilde{Y}$ is Borel. This is based on the following result from descriptive set theory. 
Theorem 2.3. Let $\mathscr{X}, \mathscr{Y}$ be Polish spaces, $P \subseteq \mathscr{X} \times \mathscr{Y}$ Borel and put

$$
x \in Q \Leftrightarrow P_{x} \text { is not meager. }
$$

Then $Q$ is Borel as well.

Using this we compute that the function $n: X \rightarrow \mathbb{N}$ is Borel and therefore $\tilde{Y}$ is Borel:

$$
n(x)=n \Leftrightarrow X_{n} \cap[x] \notin J_{[x]_{E}} \wedge \forall m<n\left[X_{m} \cap[x] \in J_{[x]_{E}}\right] .
$$

Since $X_{n} \cap[x]=\left\{y \in[x]_{E}: y \in X_{n}\right\}$, it is clearly enough, by the definition in $\S 1$ (II), to show that the assignment $C \mapsto J_{C}$ is Borel. So fix $A \subseteq X^{2}$ Borel. Then

$$
x \in A_{J} \Leftrightarrow\left\{y \in[x]_{E}: A(x, y)\right\} \in J_{[x]_{E}} \Leftrightarrow\{g: A(x, x g)\} \text { is meager }
$$

so $A_{j}$ is Borel by the preceding theorem.

Let us notice now some further facts about $\tilde{Y}$.

(1) $R \mid \tilde{Y}$ is an equivalence relation: This is because if $x, y, z \in \tilde{Y}$ are such that $R(x, y), R(y, z)$ then $z, y, z \in X_{n(x)}$ (and $n(x)=n(y)=n(z)$ ), so $R(x, z)$ holds.

(2) $R \mid \tilde{Y}$ is smooth: Because if $f_{n}: X_{n} \rightarrow Z_{n}$ witness the smoothness of $R \mid X_{n}$, with $Z_{n}$ pairwise disjoint, then $f(x)=f_{n(x)}(x), f: \tilde{Y} \rightarrow \bigcup_{n} Z_{n}$ witnesses the smoothness of $R \mid \tilde{Y}$.

(3) If $Z \subseteq \tilde{Y}$ is a transversal for $R \mid \tilde{Y}$, i.e. it meets every $R \mid \tilde{Y}$-equivalence class in exactly one point, then $Z$ is a complete lacunary section for $E$ : This is because $\tilde{Y}$ meets every $E$-equivalence class and if $(x, y) \notin R$ then $y \notin x \Delta$.

(4) $\tilde{Y}$ meets every $E$-equivalence class and there are only countably many $R \mid \tilde{Y}$ equivalence classes in each $E$-equivalence class. (This follows from (3).)

Define then the following subset $Y$ of $\tilde{Y}$ :

$$
x \in Y \Leftrightarrow x \in \tilde{Y} \wedge[x]_{R \mid \tilde{Y}} \notin J_{[x]_{E}}
$$

Clearly $Y$ is Borel and has properties (1)-(3) (with $\tilde{Y}$ replaced there by $Y$ ). But moreover it has the following further property

(5) $D \in Y /(R \mid Y) \Rightarrow D \notin J_{[D]_{\epsilon}}$.

We can define then a Borel assignment $D \mapsto I_{D}$ for $D \in Y /(R \mid Y)$ by

$$
B \in I_{D} \Leftrightarrow B \in J_{[D]_{E}}
$$

(To see that it is Borel notice that for $A \subseteq Y^{2}$

$$
\begin{aligned}
x \in A_{I} & \Leftrightarrow\left\{y \in[x]_{R \mid Y}: A(x, y)\right\} \in I_{[x]_{R \mid Y}} \\
& \left.\Leftrightarrow\left\{y \in[X]_{E}: y \in Y \wedge y R x \wedge A(x, y)\right\} \in J_{[x]_{E}} .\right)
\end{aligned}
$$

So the proof will be complete once we establish the following key fact which comes essentially from Theorem 4.1.1 of [Ke1].

THEOREM 2.4. Let $F$ be a smooth Borel equivalence relation on a standard Borel space $Y$. Assume there is a Borel assignment $D \mapsto I_{D}$ of $\sigma$-ideals to each equivalence class $D \in Y / F$. Then there is a Borel transversal $Z$ for $F$.

Proof. Without loss of generality we can assume that $Y$ is a Borel subset of a Polish space $Y$. Recall now the following standard result of descriptive set theory.

TheOREM 2.5. If $\mathscr{X}$ is Polish, $H \subseteq \mathscr{X}$ a Borel set, then there is closed $G \subseteq \mathcal{N}\left(=\mathbb{N}^{\mathbb{N}}\right.$, the Baire space) and continuous injective $\pi: G \rightarrow \mathscr{X}$ with $\pi[G]=H$.

This has the following immediate corollary. 
Corollary 2.6. Let $H$ be a Borel set in a Polish space $\mathscr{X}$. Then there is a family $\left\{H_{s}\right\}$, where $S$ varies over $\mathbb{N}^{<\omega}$ (the set of finite sequences of natural numbers), such that

(i) $H_{s}$ is Borel;

(ii) $H_{\varnothing}=H ; H_{s^{\wedge} n} \cap H_{s^{*} m}=\varnothing$, if $n \neq m ; H_{s}=\bigcup_{n} H_{s^{\wedge} n}$;

(iii) If $\alpha \in \mathcal{N}$ and $H_{\alpha \mid n} \neq \varnothing$ for all $n$, then $H_{\alpha}:=\bigcap_{n} H_{\alpha \mid n}$ is a singleton $\{x\}$ and for any $x_{n} \in H_{\alpha \mid n}, x_{n} \rightarrow x$.

Proof (of corollary). Let $G, \pi$ be as in the preceding theorem. For $x \in \mathbb{N}^{<\omega}, \gamma \in \mathcal{N}$ let $s \subset \gamma$ iff $s$ is a initial segment of $\gamma$. Put

$$
x \in H_{s} \Leftrightarrow s \subset \pi^{-1}(x) .
$$

Then (i), (ii) are obvious. For (iii), fix $\alpha$ with $H_{\alpha \mid n} \neq \varnothing$ for all $n$. Let $x_{n} \in H_{\alpha \mid n}$. Then $\alpha \mid n \subset \pi^{-1}\left(x_{n}\right)=\alpha_{n} \in G$. So $\alpha_{n} \rightarrow \alpha \in G$ and therefore $\pi\left(\alpha_{n}\right)=x_{n} \rightarrow \pi(\alpha)=x$. Since $\alpha \mid n \subset \alpha=\pi^{-1}(x), x \in H_{\alpha}$.

We complete now the proof of Theorem 2.4. Let $f: Y \rightarrow \mathscr{W}, \mathcal{W}$ Polish, be a Borel function with $y F z \Leftrightarrow f(y)=f(z)$. Put

$$
H(y, w) \Leftrightarrow f(y)=w
$$

so that $H$ is Borel (in $\mathscr{X}=Y \times \mathscr{W}$ ). Let $\left\{H_{s}\right\}$ be the family of the preceding corollary. For each $w \in$ range $(f)$, put $H_{s}^{w}=\left\{y: H_{s}(y, w)\right\}$. Then $\left\{H_{s}^{w}\right\}$ satisfies (i)-(iii) of the preceding corollary for $H^{w}:=H_{\varnothing}^{w}=f^{-1}(w)$ (which is a $F$-equivalence class). Since $H_{(n)}^{w}$ is a partition of $H_{\varnothing}^{w}$ and $I_{H^{w}}$ is a $\sigma$-deal (with $H^{w} \notin I_{H^{w}}$ ), find the least $n$ with $H_{(n)}^{w} \notin I_{H^{w}}$ and call it $\alpha^{w}(0)$. Since now $H_{\left(\alpha^{\prime \prime}(0), n\right)}^{w} \notin I_{H^{w}}$ is a partition of $H_{\left(\alpha^{w}(0)\right)}^{w}$, find the least $n$, call it $\alpha^{w^{\prime}}(1)$, with $H_{\left(\alpha{ }^{\prime \prime}(0), n\right)}^{w} \notin I_{H^{w}}$, etc. Clearly $H_{\alpha{ }^{\prime \prime} \mid n}^{w} \notin I_{H^{w}}$ for all $n$, so $H_{\alpha}^{w}$ is a singleton, say $y(w)$. So we have chosen for each $w \in \operatorname{range}(f)$ an element $y(w) \in f^{-1}(w)$. Clearly if

$$
Z=\{y(w): w \in F[Y]\}
$$

$Z$ is a transversal for $F$. It remains to show that $Z$ is Borel. We have

$$
y \in Z \Leftrightarrow y=y(f(y)) \Leftrightarrow \forall n\left(y \in H_{\alpha^{f(y)} \mid n}^{f(y)}\right) .
$$

So it is enough to check that for each $n$ the set

$$
Z_{n}=\left\{y: y \in H_{\alpha^{(y)} \mid n}^{f(y)}\right\}
$$

is Borel. First notice that if for each $y$ we define $n_{0}(y), n_{1}(y), \ldots$ to be the unique integers such that

$$
y \in H_{\left(n_{0}(y), \ldots, n_{k}(y)\right),}^{f(y)} \text { for all } k
$$

then $n_{i}: Y \rightarrow \mathbb{N}$ are Borel functions. We proceed now to show that $Z_{n}$ is Borel by induction on $n$ :

For $n=0: Z_{0}=\left\{y: y \in H_{\varnothing}^{f(y)}\right\}=Y$.

For $n=1: y \in Z_{1} \Leftrightarrow H_{\left(n_{0}(y)\right)}^{f(y)} \notin I_{[y]_{F}} \wedge \forall n<n_{0}(y)\left[H_{(n)}^{f(y)} \in I_{[y]_{F}}\right]$.

Since

$$
H_{(n)}^{f(y)} \in I_{[y]_{F}} \Leftrightarrow\left\{x:(x, f(y)) \in H_{(n)}\right\} \in I_{[y]_{F}}
$$

and the assignment $D \mapsto I_{D}$ is Borel, it is clear that $Z_{1}$ is Borel.

For $n=2: y \in Z_{2} \Leftrightarrow y \in Z_{1} \wedge H_{\left.\left(n_{1}\right)(y), n_{1}(y)\right)}^{f(y)} \notin I_{[y]_{F}} \wedge \forall n<n_{1}(y)\left[H_{\left(n_{0}(y), n\right)}^{f(y)} \in I_{[y]_{F}}\right]$, so again $Z_{2}$ is Borel.

Proceed this way ad infinitum... 
Remarks. (i) Theorem 2.4 is a generalization of the result in Burgess [B], with a different proof. In [B], the author proves that if $F$ is a smooth Borel equivalence relation in a Polish space $X$ and $F$ is induced by the continuous action of a Polish group on $X$, then $F$ has a Borel transversal. This follows from 2.4 by $\S 1$ (II), example (i).

(ii) It was mentioned in $\S 1$ (II) that there is a Borel equivalence relation $\mathrm{E}$ reducible to a countable Borel $F$ but which is not induced by a Borel action of a second countable locally compact group $G$. In fact, we can see that there is smooth Borel $E$ which is not induced by a Borel action of a Polish group: Let $A \subseteq \mathcal{N}$ be analytic but non-Borel and let $H \subseteq \mathcal{N} \times \mathcal{N}$ be closed with $A=\operatorname{proj}[H]$. Define on $H$ the equivalence relation $E$ by $(x, y) E\left(x^{\prime}, y^{\prime}\right) \Leftrightarrow x=x^{\prime}$. This is clearly Borel (in fact closed) smooth but cannot have a Borel transversal. Otherwise if $Z$ was such a transversal, $A=\operatorname{proj}[Z]$ and for all $x \in A$ there is unique $y$ with $(x, y) \in Z$, so $A$ would be Borel, a contradiction.

\section{Proof of Theorem 1.5}

We show first that (i) $\Rightarrow$ (ii). Say $E$ has a complete countable Borel section $Y$. Put $F=E \mid Y$. Thus $F$ is countable and $E \leq F$ because the relation $S(x, y) \Leftrightarrow x E y \wedge y \in Y$ is Borel and for each $x, S_{x}=\{y: S(x, y)\}$ is countable and non- $\varnothing$, so by a standard uniformization theorem there is a Borel function $f: X \rightarrow Y$ with $x E f(x)$. Thus $f$ reduces $E$ to $F$. Now any countable Borel equivalence relation $F$ can be written as $\bigcup_{n} F_{n}$ with $F_{n}$ smooth Borel equivalence relations. To see this notice that in Feldman-Moore [FM], it is shown that if $F$ is a countable Borel equivalence relation on $Y$ then there is a sequence $\left\{g_{n}\right\}$ of idempotent Borel automorphisms of $Y$ with $x F y \Leftrightarrow \exists n\left(g_{n}(x)=y\right)$. So put $x F_{n} y \Leftrightarrow x=y \vee g_{n}(x)=y$. Then each equivalence class of $F_{n}$ has cardinality $\leq 2$, thus $F_{n}$ is smooth. Let now $E_{n}=f^{-1}\left[F_{n}\right]$, i.e. $x E_{n} y \Leftrightarrow$ $f(x) F_{n} f(y)$. Then $E=\bigcup_{n} E_{n}$ and $E_{n}$ is smooth. So we proved (a) of (ii). To prove (b) we actually prove $\left(b^{\prime}\right)$ of (iii), which is clearly stronger (given $x \mapsto \mu_{x}$ define $I_{C}$ by $A \in I_{C} \Leftrightarrow \mu_{x}(A)=0$, for any $x \in C$.)

Consider again the relation $S(x, y) \Leftrightarrow x E y \wedge y \in Y$ introduced above. As its sections are countable we have by a standard result in descriptive set theory a countable sequence $f_{n}$ of Borel functions, $f_{n}: X \rightarrow Y$, with $\left\{f_{n}(x): x \in X\right\}=S_{x}=Y \cap[x]_{E}$. Let $\delta_{z}$ denote the Dirac measure at the point $z$ and finally put $\mu_{x}=\sum 1 / 2^{n+1} \cdot \delta_{f_{n}(x)}$. This clearly works.

We have just seen that also (i) $\Rightarrow$ (iii) and (iii) $\Rightarrow$ (ii). So it only remains to prove that (ii) $\Rightarrow$ (i).

First notice that $\forall x \exists n\left([x]_{E_{n}} \notin I_{[x]_{E}}\right)$. This is because $[x]_{E}=\bigcup_{n}[x]_{E_{n}}$. Let

$$
X_{n}=\left\{x:[x]_{E_{n}} \notin I_{[x]_{E}}\right\} \text {. }
$$

Thus $X=\bigcup_{n} X_{n}$. Also $X_{n}$ is $E_{n}$-invariant and Borel. Moreover for each $C \in X / E$, $C \cap X_{n}$ contains only countably many $E_{n}$-equivalence classes by the ccc of $I_{C}$. We will show that if $F_{n}=E_{n} \mid X_{n}$, then there is a Borel transversal $Y_{n} \subseteq X_{n}$ for $F_{n}$. Thus $C \cap Y_{n}$ is countable for each $C \in X / E$. Let $Y=\bigcup_{n} Y_{n}$. Then $Y$ is a countable Borel section for $E$. Since for any $C=[x]_{E}, x \in X_{n}$ for some $n$ it follows that $C \cap Y_{n} \neq \varnothing$, so $Y$ is a complete section. 
To prove the existence of $Y_{n}$ consider $\left(X_{n}, F_{n}\right)$. It is clearly a smooth Borel equivalence relation. Define a Borel assignment $D \mapsto J_{D}$ of $\sigma$-ideals to each equivalence class $D \in X_{n} / F_{n}$ by

$$
A \in J_{D} \Leftrightarrow A \in I_{[D]_{E}} .
$$

Then by Theorem 2.4 we are done.

\section{Proof of Theorem 1.6}

The implications (i) $\Rightarrow$ (ii) $\Rightarrow$ (iii) $\Rightarrow$ (v) and (ii) $\Rightarrow$ (iv) $\Rightarrow$ (v) are clear. So it is enough to prove $(\mathrm{v}) \Rightarrow(\mathrm{i})$. The proof will be based on the following

THEOREM 4.1. Let $G$ be a standard Borel group acting in a Borel way on a standard Borel space $X$ with $E_{G}$ the corresponding equivalence relation. Assume

(i) The action is free (i.e. $x g \neq x$, if $x \in X, g \neq 1$ ),

(ii) There is a probability measure $\nu$ on $X$ which is $G$-quasi-invariant,

(iii) there is an assignment $x \mapsto \mu_{x}$ with $\mu_{x}\left([x]_{E_{G}}\right)=1$ and $x E_{G} y \Rightarrow \mu_{x} \sim \mu_{y}$, which is $\nu$-measurable, i.e. for each bounded Borel $B: X^{2} \rightarrow \mathbb{R}$ the function $b: X \rightarrow \mathbb{R}$ given by $b(x)=\int_{[x]_{E}} B(x, y) d \mu_{x}(y)$, where $E=E_{G}$, is $\nu$-measurable.

Then $G$ is Polish locally compact.

Proof. Define the following probability measure on $G$ : For $A \subseteq G$ Borel, let

$$
\mu(A)=\int \mu_{x}(x A) d \nu(x) .
$$

The function $x \mapsto \mu_{x}(x A)$ is $\nu$-measurable, so that this integral makes since. This is because

$$
\mu_{x}(x A)=\int_{[x]_{E}} B(x, y) d \mu_{x}(y)
$$

where $B(x, y)=1$, if $y \in x A$; =0 if $y \notin x A$. As the action is free, the relation

$$
P(x, y) \Leftrightarrow y \in x A \Leftrightarrow \exists g[g \in A \wedge y=x g] \Leftrightarrow \exists ! g[g \in A \vee y=x g]
$$

is Borel, so $F$ is Borel. (That $\mu$ is countably additive follows also from the freeness of the action.)

We check now that $\mu$ is (left) quasi-invariant. i.e. $\mu \sim g \mu$ for all $g \in G$. Let $\mu(A)=0$. we will show that $\mu(g A)=0$. Since

$$
\mu(A)=\int \mu_{x}(x A) d \nu(x)
$$

clearly $\mu_{x}(x A)=0 \nu$-a.e. Now

$$
\left.\mu(g A)=\int \mu_{x}(x(g A)) d \nu(x)=\int \mu_{x}((x g) A)\right) d \nu(x)=\int \mu_{x y-1}(x A) \frac{d(\nu g)}{d \nu}(x) d \nu(x)
$$

by the $G$-quasi-invariance of $\nu$. But $\mu_{x}(x A)=0 \Rightarrow \mu_{x g-1}(x A)$, as $\mu_{x} \sim \mu_{x x-1}$. So $\mu(g A)=0$.

Now a theorem of Mackey [Ma] asserts that if $G$ is a standard Borel group which admits a quasi invariant probability measure, then $G$ is Polish locally compact and our proof is complete. 
Assume now (v) in Theorem 1.6. By the preceding theorem it is enough to find the assignment $x \mapsto \mu_{x}$ for $E^{0}=E_{G}^{0}$. Say $F$ is a countable Borel equivalence relation on $Y$ and $f: X_{0} \rightarrow Y$ reduces $E^{0}$ to $F$. The relation $R(x, y) \Leftrightarrow y=f(x)$ is Borel, so there is $C$-measurable $g: f\left[X_{0}\right] \rightarrow X_{0}$ with $f(g(y))=y$, where $C$ is the smallest $\sigma$-algebra containing the Borel sets and closed under the Souslin operation $\mathscr{A}$. As $F$ is countable we can find, by Feldman-Moore [FM], a countable group $\left\{g_{n}\right\}$ of Borel automorphisms of $Y$ inducing $F$. Put $f_{n}(x)=g\left(g_{n}(f(x))\right.$. Then $\left\{f_{n}(x): n \in \mathbb{N}\right\}=$ $g\left[[f(x)]_{F}\right] \subseteq[x]_{E_{0}}$ depends only on $[x]_{E_{0}}$. Put $\mu_{x}=\sum 1 / 2^{n+1} \cdot \delta_{f_{n}(x)}$. Clearly $\mu_{x}\left([x]_{E^{0}}\right)=1$ and $x E^{0} y \Rightarrow \mu_{x} \sim \mu_{y}$. Finally $x \mapsto \mu_{x}$ is $\nu$-measurable, since each $f_{n}$ is $C$-measurable, so if $B: X_{0}^{2} \rightarrow \mathbb{R}$ is bounded Borel, then $b(x)=\int B(x, y) d \mu_{x}(y)=$ $\sum 1 / 2^{n+1} . B\left(x, f_{n}(x)\right)$ is also $C$-measurable, thus $\nu$-measurable.

\section{Some examples}

We consider first the equivalence realtion $E_{1}$ on $\mathbb{T}^{\mathbb{N}}$ given by

$$
\left(x_{n}\right) E_{1}\left(y_{n}\right) \Leftrightarrow \exists m \forall n \geq m\left(x_{n}=y_{n}\right) \text {. }
$$

We will give a proof that $E_{1}$ is not reducible to a countable Borel equivalence relation based on Theorem 1.6 (and the remark following it.)

Let $H=\mathbb{T}^{\mathbb{N}}, G=\bigcup_{n} \mathbb{T}^{n}=\left\{\left(x_{n}\right) \in H: \exists m \forall n \geq m\left(x_{n}=1\right)\right\}$. Thus $H$ is a Polish compact group and $G$ is a Borel (actually $F_{\sigma}$ ) subgroup of $H$. If $E_{G}=E_{1}$ is reducible to a countable Borel equivalence relation, then $G$ would be Polish (locally compact). Then by the Baire category theorem $\mathbb{T}^{n}$ would be non-meager (in the Polish topology of $G$ ) for all large enough $n$, so $\left(\mathbb{T}^{n}\right)^{-1} \mathbb{J}^{n}=\mathbb{T}^{n}$ would have to contain an open nbhd of the identity by a standard fact on Polish groups (see for example [C]). So $\mathbb{T}^{n}$ would be open, therefore Polish (with the relative topology). But then by the continuity of Borel homomorphisms on Polish groups this must be the standard topology on $\mathbb{T}^{n}$. Thus $\mathbb{T}^{n}$ is open in $\mathbb{J}^{n+1}$ with the standard topology, which is absurd.

Our final example will be that of a $K_{\sigma}$ equivalence relation on $\mathbb{T}^{\mathbb{N}}$ which is induced by a free continuous action of a Polish group but is not reducible to a countable Borel equivalence relation.

Put again $H=\pi^{\mathbb{N}}$ and

$$
G=\left\{\left(x_{n}\right) \in H: \sum\left|1-x_{n}\right|^{2}<\infty\right\}=\left\{\left(e^{i y_{n}}\right) \in H: \sum \sin ^{2}\left(y_{n} / 2\right)<\infty\right\} .
$$

We verify the following facts.

Fact 1. $\sqrt{\sum \sin ^{2}\left(a_{n}+b_{n}\right)} \leq \sqrt{\sum \sin ^{2}\left(a_{n}\right)}+\sqrt{\sum \sin ^{2}\left(b_{n}\right)}$.

Proof. Assume without loss of generality that the sums are finite and square both sides.

Fact 2. $G$ is a subgroup of $H$.

Proof. Immediate from Fact 1.

Fact 3. $G$ is $F_{\sigma}$ in $H$.

Define now the metric $d$ on $G$ by

$$
d\left(\left(e^{i y_{n}}\right),\left(e^{i u_{n}}\right)\right)=\sqrt{\sum \sin ^{2}\left(\left(y_{n}-u_{n}\right) / 2\right.}+\sum 2^{-n}\left|e^{i y_{n}}-e^{i u_{n}}\right| .
$$

(That it is a metric follows from Fact 1.) Clearly the identity map on $G$ is continuous from $(G, d)$ into $H$. 
Fact 4. $(G, d)$ is a Polish group.

Proof. Since $d$ is translation invariant $(G, d)$ is a metric group. It is separable since eventually 1 sequences with rational coordinates are dense in $(G, d)$. Completeness is checked easily, the argument being similar to that showing completeness of $l^{2}$.

Consider now $E_{G}$ induced by the action of $G$ on $H$. It is clearly $K_{\sigma}$ and induced by a free continuous action of $(G, d)$. By Theorem 1.6 , if $E_{G}$ was reducible to a countable Borel equivalence relation, then $(G, d)$ would be locally compact.

Fact $5 .(G, d)$ is not locally compact.

Proof. Let $U=\left\{\left(x_{n}\right): d\left(1,\left(x_{n}\right)\right)<\varepsilon\right\}$ be a nbhd of 1 . It suffices to find $\left(x_{n}^{i}\right)$, a sequence in $U$, which has no converging subsequence. For that it is enough to have $d\left(\left(x_{n}^{i}\right)\right.$, $\left.\left(x_{n}^{j}\right)\right)>\delta$ for $i \neq j$ and some $\delta>0$. Put $\left(x_{n}^{i}\right)=\delta_{i, n} e^{i \theta}$, where $\delta$ is the Kronecker delta and $\theta>0$. For $\theta$ small enough

$$
d\left(1,\left(x_{n}^{i}\right)\right)=\sqrt{\sin ^{2}(\theta / 2)}+2^{-i}\left|1-e^{i \theta}\right|<\varepsilon
$$

and for some $\delta>0$

$$
d\left(\left(x_{n}^{i}\right),\left(x_{n}^{j}\right)\right)=\sqrt{2 \sin ^{2}(\theta / 2)}-\left(2^{-i}+2^{-j}\right)\left|1-e^{i \theta}\right|>\delta .
$$

\section{REFERENCES}

[A] W. Ambrose. Representation of ergodic flows. Ann. of Math. 42 (1941), 723-739.

[B] J. Burgess. A selection theorem for group actions. Pac. J. Math. 80 (1979), 333-336.

[C] J. P. R. Christensen. Topology and Borel Structure. (North-Holland, Amsterdam, 1974).

[FHM] J. Feldman, P. Hahn \& C. C. Moore. Orbit structure and countable sections for actions of continuous groups. Adv. Math. 26 (1979), 186-230.

[FM] J. Feldman \& C. C. Moore. Ergodic equivalence relations, cohomology and von Neumann algebras, I. Trans. Amer. Math. Soc. 234 (1977), 289-324.

[FR] J. Feldman \& A. Ramsay. Countable sections for free actions of groups. Adv. Math. 55 (1985), 224-227.

[F] P. H. Forrest. Virtual subgroups of $\mathbb{R}^{n}$ and $\mathbb{Z}^{n}$. Adv. Math. 3 (1974), 187-207.

[HKL] L. Harrington, A. S. Kechris \& A. Louveau. A Glimm-Effros dichotomy for Borel equivalence relations. J. Amer. Math. Soc. 3 (1990), 903-928.

[Ke1] A. S. Kechris. Measure and category in effective descriptive set theory. Ann. Math. Logic 5 (1973), 337-384.

[Ke2] A. S. Kechris. The structure of Borel equivalence relations in Polish spaces. Set Theory and the Continuum. H. Judah, W. Just and W. H. Woodin, eds, MSRI Publications, Springer-Verlag, to appear.

[Ku] K. Kuratowski. Topology. Vol. I (Academic Press: New York, 1966).

[Ma] G. W. Mackey. Borel structures in groups and their duals. Trans. Amer. Math. Soc. 85 (1957), 134-165.

[Mi] D. Miller. On the measurability of orbits in Borel actions. Proc. Amer. Math. Soc. 63 (1977), 165-170.

[MZ] D. Montgomery \& L. Zippin. Topological Transformation groups (Interscience: New York, 1955).

[Mo] Y. N. Moschovakis. Descriptive Set Theory. (North-Holland: Amsterdam, 1980).

[R1] A. Ramsay. Topologies on measured groupoids. J. Funct. Anal. 47 (1982), 314-343.

[R2] A. Ramsay. Local product structure for group actions. Ergod. Th. \& Dynam. Sys. 11 (1991), 209-217.

[Var] V. S. Varadarajan. Groups of automorphisms of Borel spaces. Trans. Amer. Math. Soc. 109 (1963), 191-220.

[Vau] R. L. Vaught. Invariant sets in topology and logic. Fund. Math. 82 (1974), 269-283.

[W] V. M. Wagh. A descriptive version of Ambrose's representation theorem for flows. Proc. Ind. Acad. Sci. (Math. Sci.) 98 (1988), 101-108. 